\title{
Transient and sustained masking
}

\author{
D. MITOV, A. VASSILEV, and V. MANAHILOV \\ Institute of Physiology, Bulgarian Academy of Sciences, 1113 Sofia, Bulgaria
}

\begin{abstract}
The detection threshold of a brief test stimulus was measured as a function of the onset asynchrony between it and a long-lasting suprathreshold masking stimulus. Both stimuli were sine-wave gratings of the same vertical orientation and in the peak-subtract phase but differed in spatial frequency by a factor of 3 . The temporal masking functions obtained with 2- and 6-cycles/deg maskers of high contrast exhibited transient on- and off-peaks of masking and a sustained effect during the masker exposure. An 18-cycles/deg masker caused sustained masking only. Experiments with maskers of variable spatial frequency and contrast showed that, in the low-spatial-frequency range, the mechanism responsible for the transient effect was more sensitive than that generating the sustained effect, while the sustained effect required less contrast in the high-spatial-frequency range. The results are considered as evidence, in addition to previous findings, for the sustained/transient dichotomy in the temporal domain.
\end{abstract}

Evidence from several types of psychophysical experiments suggests that the response of the visual system to a stimulus of high spatial frequency is sustained or predominantly sustained; that is, a level of activity is maintained throughout the stimulus exposure, whereas the response to a stimulus of low spatial frequency is transient, with peaks of activity at the stimulus onset and offset. Thus, at threshold contrast, the detection of coarse gratings depends on the activity related to their onset or offset as revealed by the effects of stimulus temporal waveform on the contrast sensitivity (Breitmeyer \& Julesz, 1975) or on the reaction time distribution (Tolhurst, 1975a). According to the same experiments, the detection of fine gratings can be related to any instant of stimulus exposure. Furthermore, masking at the onset and the offset of long-lasting test stimuli is effective only if the test stimulus spatial frequency is low (Legge, 1978). Different temporal summation paradigms also yield results characteristic of transient activity at low spatial frequency and of sustained activity at high spatial frequency (Legge, 1978; Lupp, Hauske, \& Wolf, 1978; Tolhurst, 1975b; Watson \& Nachmias, 1977). This dependence of time course of response on stimulus spatial frequency seems to hold also for gratings that are well above the threshold, as indicated by the changes in apparent contrast with stimulus duration (Kitterle \& Corwin, 1979).

On the other hand, it has been known since the work of Crawford (1947) that a brief test spot superimposed on a long-lasting masking disk has the highest threshold of detection at or near the time interval of the masker onset. A second peak of masking is

Part of this paper was reported at the Third European Conference on Visual Perception, Brighton, England, September 3-6, 1980. sometimes found at the masker offset. The conditions known to be necessary for the existence of such peaks of masking are a high intensity of the masker and a large masker area (Teller, Matter, Phillips, \& Alexander, 1971). Boynton (1961) attributed the ontransient of masking to the initial burst of activity caused by the masker onset. Most data on temporal masking (for reviews, see Sperling, 1965, and Teller et al., 1971) are in agreement with such an interpretation.

All these data, considered together, suggest definite effects of the masker spatial frequency on the time course of masking: At low spatial frequency, masking will exhibit transient on- and off-peaks; at high spatial frequency, masking will be predominantly sustained.

Earlier works of ours (Mitov, 1979; Mitov, Nevskaya, \& Vassilev, 1980) have shown this to be the case. The detection threshold of a test grating was mostly elevated at or near the instants of switching on and off of a low- or medium-spatial-frequency masker grating. It was, however, maintained at an almost constant level, higher than the unmasked threshold, throughout the exposure of a high-spatial-frequency masker. This finding is confirmed by Experiment 1 , presented below. The subsequent experiments extend the initial studies and allow inferences about the contrast sensitivity relationship of the mechanisms generating the transient and sustained components of masking.

\section{METHODS}

\footnotetext{
Apparatus

Both test and masking stimuli were sine-wave gratings. They were generated on the screen of an RFT FB1 TV monitor used with standard sweep and frame rates (625 lines and 50 fields/sec). The signal generating one of the gratings was the output of a sine-
} 
wave oscillator. It was triggered by the TV sweep generator in order to stabilize the picture on the monitor. A second sine-wave signal, synchronized with the first, was obtained from the same output of the oscillator by the following operations: conversion to a square-wave by a Smitt trigger, subtraction of the undistorted sine-wave, and active filtering to obtain the third harmonic. Because of this peculiarity of stimulus generation, the gratings were always in the 3:1 spatial frequency ratio (other ratios, including $1: 1$, could also be obtained, but they have not yet been utilized). The relative phase angle between test and masking gratings was zero (peak subtract phase). The amplitude of each sine-wave signal was monitored independently by an attenuator. Linearity between this amplitude and grating contrast, as measured with a photomultiplier aimed at the screen of the monitor, held up to a contrast of .7. The contrast, $m$, was defined as $m=\left(L_{\max }-L_{\text {min }}\right)$ ' $\left(\mathrm{L}_{\max }+\mathrm{L}_{\min }\right)$. The light emitted by the luminescent layer was white. The mean luminance of the screen was constant, did not change on presenting the gratings, and was equal to $30 \mathrm{~cd} / \mathrm{m}^{2}$.

The viewing distance was $138 \mathrm{~cm}$. A mask on the screen limited the stimulus field to a circle, subtending 3 deg of visual angle in diameter at the subject's eyes. The surrounding field was dark. Viewing was binocular, with natural pupils and accommodation.

\section{Procedure}

The threshold contrast necessary for the detection of the test stimulus was measured by the two-interval forced-choice method combined with the staircase procedure. To this end, on each trial, the masker was presented twice with a blank interval of $1 \mathrm{sec}$ and the test stimulus was presented randomly with either the first or the second exposure. The subject's task was to determine which masker exposure was accompanied by the test grating. The duration of the test stimulus was $20 \mathrm{msec}$, while that of the masker was $500 \mathrm{msec}$. The following staircase procedure was adopted: The contrast of the test stimulus was increased by .1 log units after a wrong answer and was decreased by the same step after three consecutive correct answers. The procedure continued until the accumulation of 10 reversals. Six to eight thresholds were measured in a daily session at different stimulus onset asynchronies (SOAs). Each measurement was repeated three times (twice in Experiment 3). Geometrical means and $95 \%$ confidence intervals were calculated on the basis of 30 ( 20 in Experiment 3 ) reversals.

The threshold of the test grating presented alone was measured by the same procedure, but, in this case, the time intervals were marked by sounds.

The trials were initiated by the subject after a "ready" signal, and there was feedback about the correctness of each answer. The subjects were instructed to look at the center of the screen. A fixation mark was used in Experiment 3 only.

\section{Subjects}

One of the authors (D.M.) and four naive male subjects, aged between 25 and 35 years and with acuity of each eye of more than 5/5 took part in the different experiments. Each subject was given two or three training sessions.

\section{Abbreviation Used in the Text}

In the text below, the stimuli are usually denoted as F2, F6, and so on, each symbol signifying a sine-wave grating of the spatial frequency (in cycles/deg) denoted by the figure.

\section{RESULTS}

\section{Experiment 1}

In this experiment, temporal masking functions were obtained with four different pairs of masking and test stimuli: F2 and F6, F6 masking F2, F6 masking F18, and F18 masking F6 (see Figure 1). The contrast of the masking grating was .5 , that is, the al-

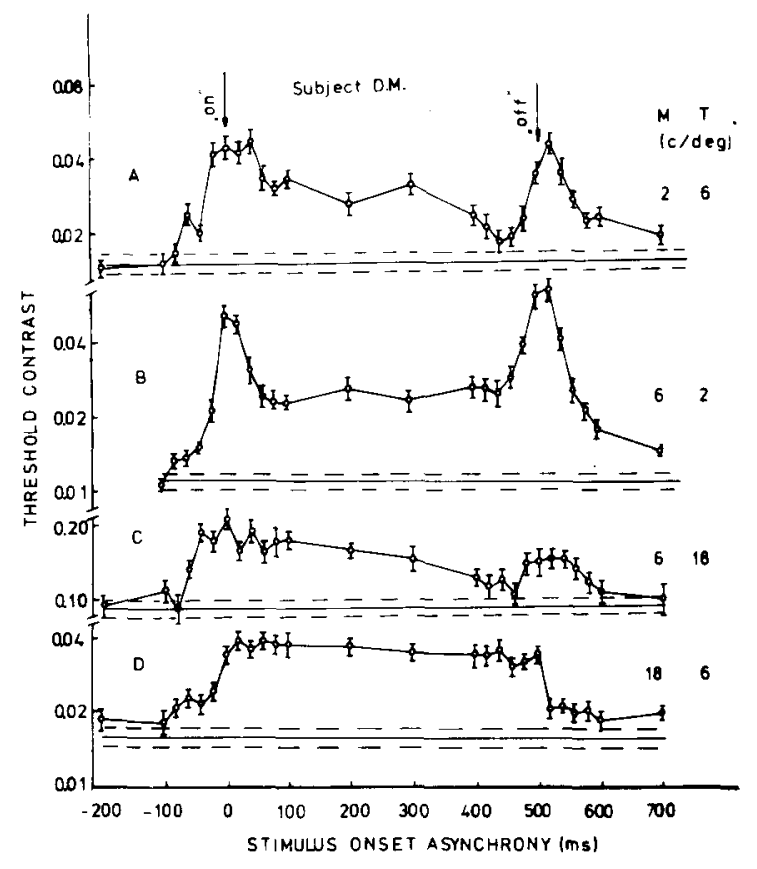

Figure 1. Temporal masking functions for different pairs of test and masking gratings. The figures on the right of each curve show the spatial frequencies of the masking (M) and test (T) stimuli. Test stimulus duration $=20 \mathrm{msec}$; masking stimulus duration $=500 \mathrm{msec}$. The arrows indicate the onset and of fset of the masker. The horizontal whole lines and the flanking dashed lines show the mean and the $95 \%$ confidence interval of the threshold for the unmasked test grating. Vertical bars show the $95 \%$ confidence interval of the experimental points. (Subject D.M.)

most maximum contrast available in the linear range of the monitor.

As can be seen, the magnitude and time course of masking were not the same with all pairs of test and masking stimuli. Curves $\mathbf{A}$ and $\mathbf{B}$ exhibit peaks of threshold at the onset and offset of the masker. Although much reduced in amplitude, such peaks are also present in Curve $C$. These peaks will be denoted as transient components of masking because of their obvious relationship to the transients of stimulation by the masker. In addition to its transient components, Curve B shows a steady threshold elevation during the masker exposure that suggests the presence of maintained masking activity and is called sustained masking.

After the on-transient, Curves $\mathrm{A}$ and $\mathrm{C}$ exhibit a slow decline of masking and an abrupt threshold drop 40-60 msec before the masker offset. This time course of masking was regularly seen if the spatial frequency of the test stimulus was higher than that of the masker. Experiments with maskers of variable exposure time proved that the abrupt threshold drop was time-locked to the masker offset (Mitov et al., 1980).

The lowest curve in Figure 1 exhibits sustained masking only. It has no transient peaks to exceed the 
sustained masking level. Note that this curve was obtained with F18 as a masker, that is, with a masker of high spatial frequency, while the other curves were obtained with maskers of lower spatial frequency.

As a rule, masking was asymmetrical. Thus, F6 was a stronger masker of $F 2$ than $F 2$ was of $F 6$ for most SOAs tested. Such asymmetrical effects have previously been found in experiments on simultaneous masking (Sansbury, 1974). The present data show that this asymmetry is particularly pronounced toward the end of the masker exposure. An exception to the above rule is the lesser degree of magnitude of masking of $\mathrm{F} 6$ by $\mathrm{F} 18$ than the magnitude of masking of F18 by F6 at SOAs near the time interval of the masker offset. The possible explanation is the lack of off-response to $\mathrm{F} 18$.

D.M. was the only observer to participate in experiments with all four combinations of test and masking gratings. His data were verified with two more subjects, each of whom, however, was presented with only two of the four combinations. Their results were similar to those of D.M. concerning the magnitude and time course of masking.

\section{Experiment 2}

The lack of transients with F18 as a masker might result from the low contrast sensitivity at this spatial frequency. Thus, we considered it reasonable to study the effect of the masker contrast on the most typical transient-sustained curve (F6 masking F2) and

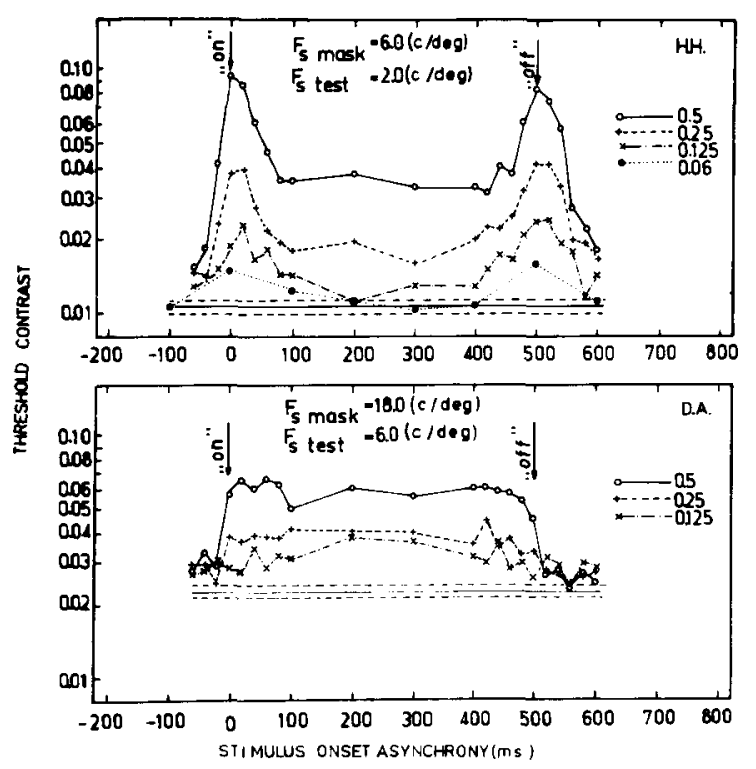

Figure 2. Effect of masker contrast on the temporal masking function. The spatial frequencies and contrast of the stimuli are given in the insert. The confidence intervals of the masked thresholds are omitted; they varied between .03 and .1 log units. (Upper graph: Subject H.H.; lower graph: Subject D.A.) on the most typical sustained curve (F18 masking F6).

Masking functions were obtained for the following contrast values of the masker: .06 (with F6 only), $.125, .25$, and .5 . The results are presented in Figure 2 . It can be seen that, for both maskers, the reduction of contrast reduces the magnitude of masking in a way that does not abolish the characteristic difference between the effects of F6 and F18. Thus, F6 preserves its transient masking effect at low contrast levels while losing its sustained effect. The time course of masking with F18 remains sustained at all contrast levels examined.

These results rule out the suggestion that the two types of temporal masking are due to contrast sensitivity factors. If masking with F18 were sustained because of low contrast sensitivity at this spatial frequency, the low-contrast F6 would have also yielded a sustained masking curve.

A delay by some $40-60 \mathrm{msec}$ of the threshold rise at the onset of low-contrast F18 was observed, compared with the initial threshold rise caused by highcontrast or low-spatial-frequency maskers. It might result from the increased latency of response to a masker grating that is of both low contrast and high spatial frequency. Both factors are known to affect the latency of response (Breitmeyer, 1975; Lupp et al., 1976; Vassilev \& Mitov, 1976).

\section{Experiment 3}

The mechanism yielding the transient masking effect of F6 in Experiment 2 was found to be of higher contrast sensitivity than the mechanism of sustained masking. The results with F18 as a masker could be interpreted as suggesting that the sustained mechanism becomes more sensitive than the transient mechanism when the stimulus spatial frequency is increased. If this is the case, it will be possible to find out a masker of spatial frequency between 6 and 18 cycles/ deg yielding both masking components at high contrast but sustained masking only at low contrast.

Experiments with two subjects were carried out. The maskers were F6 and F8.4, and the contrast was varied in order to obtain data about its effect on the peaks and plateau of the masking curves. For each masker, the spatial frequency of the test stimulus was three times lower than the masker spatial frequency.

The upper graphs in Figures 3 and 4 are for masking with F6. They show, again, that reducing the contrast of F6 eliminates its sustained masking effect, leaving only slight on-off transient threshold elevations. The lower graphs represent the time course of masking with F8.4. At the contrast of .5, masking is similar to that with F6, exhibiting both transient and sustained components. However, F8.4 loses its transient masking effect at any one of the lower contrast levels, while the sustained effect is still present-a result that is opposite to that with F6 as a masker. 


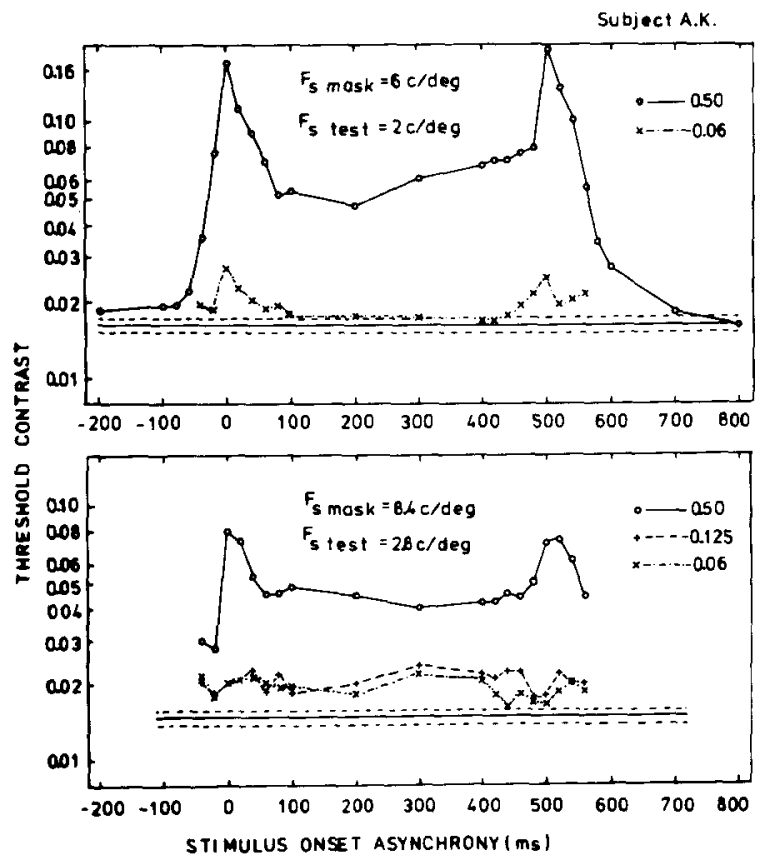

Figure 3. Effect of masker contrast on the temporal masking function. Other details as in Figures 1 and 2. (Subject A.K.)

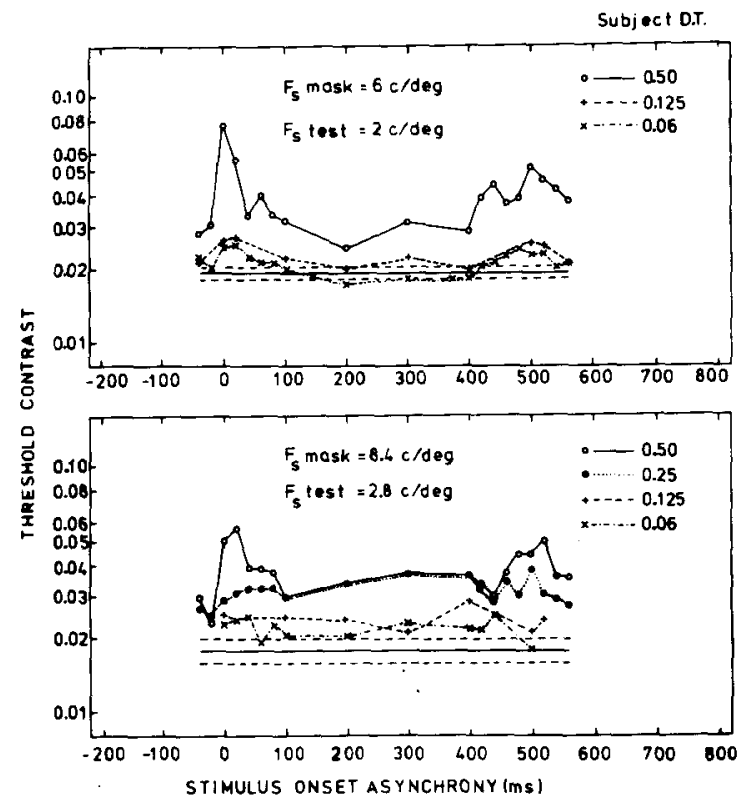

Figure 4. Same as Figure 3, but for Subject D.T.

\section{DISCUSSION}

Several authors have proposed the existence of two distinct mechanisms of human vision responsible for the recognition of temporal and spatial contrast, termed "transient"' and "sustained," respectively (Keesey, 1972; Kulikowski \& Tolhurst, 1973; Tolhurst, 1973; see also Blake, 1979, and Legge, 1978, for other references). The transient mechanism, denoted also as the "movement analyzer" (Tolhurst, 1973) has a high temporal and a low spatial frequency resolution power. The sustained mechanism, also called the "form analyzer," has a higher spatial frequency resolution power than the transient mechanism and a lower temporal one.

The studies demonstrating a transient response to gratings of low spatial frequency and a sustained response to gratings of high spatial frequency (see the introduction) are particularly relevant to the present experiments. In line with these studies, the following masking functions were found: pure transient, combined transient and sustained, and pure sustained. The first type was observed with low-contrast maskers of 6 cycles/deg. The second type was seen with highcontrast maskers in the intermediate frequency range (2-8.4 cycles/deg). The third type characterized masking with gratings of high spatial frequency, regardless of their contrast. Furthermore, depending on the masker spatial frequency, either the transient or the sustained component of masking was the first to disappear on reducing the contrast of the masking stimulus. These findings suggest that the mechanism generating transient masking and that responsible for the sustained masking have different contrast sensitivities, the first being more sensitive in the lowspatial-frequency range and the second being more sensitive in the high-spatial-frequency range. ${ }^{1}$

As explained in the introduction, one of the assumptions on which this study is based is that of the dependence of temporal masking on the time course of response to the masking stimulus. The comparison of curves obtained with different pairs of test and masking stimuli (Figure 1) allows us to reconsider this statement. It seems that the masking stimulus is the major factor as far as considering such general characteristics of the temporal masking functions as the presence or absence of transient and sustained components. However, the ratio of test-to-masker spatial frequencies and the test stimulus itself are additional factors modifying the time course of masking. Thus, the diminution of masking at SOAs preceding the masker offset was observed only if the spatial frequency of the test stimulus was higher than that of the masker. We have no explanation for this finding. The transient peaks of masking caused by the same masker, F6, were of much lower amplitude with F18 than with $\mathrm{F} 2$ as a test stimulus. ${ }^{2}$ The smearing effect of F18 was probably related to the fact that the response to a stimulus of high spatial frequency is spread over a longer time interval than the response to a low-frequency stimulus (Tolhurst, 1975a). Yet, transient peaks were still detectable with $\mathrm{F} 18$ as a test. This supports the notion of the importance of the time course of response to the masking stimulus in determining the time course of masking.

If this assumption is correct, the present data seem to be best interpreted in terms of contrast sensitivities of transient and sustained mechanisms. The pure sus- 
tained masking at high spatial frequency of the masker is explained by the insensitivity of transient mechanisms within this range. The presence of both components of masking in the intermediate frequency range results from the overlap of the contrast sensitivity functions of transient and sustained mechanisms. Such an overlap is suggested by the experiments of Legge (1978). On increasing stimulus spatial frequency, the higher contrast sensitivity of the mechanism generating transient masking is replaced by the higher sensitivity of the mechanism responsible for the sustained masking. Experiment 3 was planned on the basis of this relationship, and the results (Figures 3 and 4 ) confirmed it.

It is to be pointed out that, according to the data of Legge (1978) and Tolhurst (1975a), the transient mechanism is more sensitive than the sustained mechanism only for spatial frequencies below 1 cycle/deg. The present results suggest that the transient mechanism dominates over the sustained mechanism up to 6 cycles/deg. The difference between the estimates is rather large. However, assessments of the spatial frequency at which the sustained mechanism becomes more effective than the transient mechanism provided by other methods are closer to the present estimate (Breitmeyer \& Julesz, 1975; Kulikowski \& Tolhurst, 1973; Watson \& Nachmias, 1977; Watson, Thompson, Murphy, \& Nachmias, 1980). The discrepancy might result from differences within subjects, different sensitivities of the methods applied, and a shift of the contrast sensitivity functions of transient and sustained mechanisms by the adaptational state, by the stimulus characteristics, or by unknown experimental conditions.

The present data seem to be an example of the dependence of the temporal characteristics of response on the spatial parameters of the stimulus, a dependence that is quite typical over a wide range of experimental conditions (Breitmeyer \& Ganz, 1976) but of still-hypothetical origin.

Tolhurst (1973) suggested that human sustained and transient mechanisms are analogous to the $X$ and Y-cells, respectively, of the cat visual system (Cleland, Dubin, \& Levick, 1971; Enroth-Cugell \& Robson, 1966). The property of X-cells to give predominantly sustained response to prolonged stimulation and the property of Y-cells to respond only transiently, together with the tendency of $\mathrm{X}$-cells to respond to higher spatial frequencies than do Y-cells, would explain most psychophysical data, including these presented here. It has been emphasized, however, that the degree of transience of response of a neuron depends on stimulus size and intensity, adaptational level, and eccentricity of its receptive field (Lennie, 1980; Podvigin, 1979). This makes the time course of response an uncertain link between psychophysics and neurophysiology. Thus, it is not clear whether, for example, a high-contrast masker of 8.4 cycles/deg (Figures 3 and 4) yields transient onand off-peaks of masking because of the involvement of a structure with a transient response or because of a modulation by the intense stimulus of the response of the same structure that yields sustained masking at lower contrast.

Arend (1976) suggested that the effects of stimulus duration on the modulation transfer function, considered to be evidence for the sustained/transient dichotomy, results from eye movements. Applied to masking, the same hypothesis would be interpreted in the following way. Eye movements, shifting the masking grating over the retina, generate temporal modulations of illuminance whose frequency increases with the spatial frequency of the masker. At a high spatial frequency, the masking effect of these modulations equals the effect of the abrupt onset and offset of the masker, the result being a sustained masking curve. Unpublished data from our laboratory have shown that the difference between transient and sustained masking becomes evident even with maskers lasting for $80 \mathrm{msec}$, an exposure time that probably reduces the eye movement effect. The direct test of this hypothesis with stabilized images is, nevertheless, desirable.

There are some hints as to the possible role of the center-surround antagonism within receptive fields in the generation of transient masking. Teller et al. (1971) measured the threshold of a small test spot presented at varying SOAs against a masking disk that was either smaller or larger than the area of densensitization of Westheimer (1965). Their masking curves show transient peaks only with the large masker that presumably invaded the antagonistic surround of the retinal area tested. Keesey and Vassilev (1974) found that the sensitizing effect of the surround was at least partially mediated by mechanisms that transmit temporal changes in illuminance. However, it is impossible to discuss the center-surround hypothesis without data on the temporal and spatial organization of the interactions forming the time course of response and on the types of receptive fields involved. We think it is necessary to collect more data on the behavior of the transient and sustained components of masking before giving preference to any explanation of their origin.

\section{REFERENCES}

Arend, L. E., JR. Temporal determinants of the form of the spatial contrast threshold MTF. Vision Research, 1976, 16, 1035-1042.

Blake, R. The visual system of the cat. Perception \& Psychophysics, 1979, 26, 423-448.

Boynton, R. M. Some temporal factors in vision. In W. A. Rosenblith (Ed.), Sensory communication. New York: Wiley, 1961. 
Breitmeyer, B. Simple reaction time as a measure of the temporal response properties of transient and sustained channels. Vision Research, 1975, 15, 1411-1412.

Breitmeyer, B., \& Ganz, L. Implications of sustained and transient channels for theories of visual pattern masking, saccadic suppression, and information processing. Psychological Review, 1976, 83, 1-36.

Breitmeyer, B., \& Julesz, B. The role of on and off transients in determining the psychophysical spatial frequency response. Vision Research, 1975, 15, 411-415.

Campelel, F. W., \& Robson, J. G. Application of Fourier analysis to the visibility of gratings. Journal of Physiology, 1968, 197, 551-556.

Carter, B. E., \& Henning, G. B. The detection of gratings in narrow-band visual noise. Journal of Physiology, 1971, 219, 355-365.

Cleland, B. G., Dubin, M. W., \& Levick, W. R. Sustained and transient neurones in the cat's retina and lateral geniculate nucleus. Journal of Physiology, 1971, 217, 473-496.

Crawford, B. H. Visual adaptation in relation to brief conditioning stimuli. Proceedings of the Royal Society of London, 1947, 134B, 283-302.

Enroth-Cugeld, C., \& Robson, J. G. The contrast sensitivity of retinal ganglion cells of the cat. Journal of Physiology, 1966, $187,517-552$.

Green, M. Spatial frequency effects in masking by light. Vision Research, 1981, 21, 861-866.

KEEsEy, U. T. Flicker and pattern detection: A comparison of thresholds. Journal of the Optical Saciety of America, 1972, 62, 446-448.

KeESEY, U. T., \& VAssilev, A. Foveal spatial sensitization with stabilized vision. Vision Research, 1974, 14, 101-105.

Kitterle, F. L., \& Corwin, T. R. Enhancement of apparent contrast in flashed sinusoidal gratings. Vision Research, 1979, 19, 33-39.

Kulikowski, J. J., \& Tolhurst, D. J. Psychophysical evidence for sustained and transient detectors in human vision. Journal of Physiology, 1973, 232, 149-162.

LegGe, G. E. Sustained and transient mechanisms in human vision: Temporal and spatial properties. Vision Research, 1978, 18, 69-81.

LegGe, G. E., \& Foley, J. M. Spatial frequency masking in human vision: Dependence on contrast and frequency. Investigative Ophthalmology and Visual Science, 1979, 18, 59. (Suppl.)

Lennie, P. Parallel visual pathways: A review. Vision Research, $1980,20,561-594$.

Lupp, U., Hauske, G., \& Wolf, W. Perceptual latencies to sinusoidal gratings. Vision Research, 1976, 16, 969-972.

LuPp, U., Hauske, G., \& Wolf, W. Different systems for the visual detection of high and low spatial frequencies. Photographic Science and Engineering, 1978, 22, 80-84.

Mitov, D. Spatial-frequency channels and their interaction. In Biology 79 (Tenth National Symposium of Young Scientists: Biology Section). Sofia, Bulgaria: United Center of Biology, Bulgarian Academy of Science, 1979.

Mitov, D., Nevskaya, A., \& Vassilev, A. Transient and sustained types of masking. Acta Physiologica et Pharmacologica Bulgarica, 1980, 6, 16. (Summary)

Pantle, A., \& Se kuler, R. Size detecting mechanisms in human vision. Science, 1968, 162, 1146-1148.

Podvigin, N. F. Dynamic properties of neuronal structures in the visual system. Leningrad: Nauka, 1979 (in Russian).

SANSBURY, R. V. Some properties of spatial channels shown by pulsed simultaneous masking. Unpublished doctoral dissertation, University of Pennsylvania, 1974.
SperLing, G. Temporal and spatial visual masking: I. Masking by impulse flashes. Journal of the Optical Society of America, 1965, 55, 541-559.

Stromeyer, C. F., III, \& Julesz, B. Spatial-frequency masking in vision: Critical bands and spread of masking. Journal of the Optical Society of America, 1972, 62, 1221-1232.

Teller, D. Y., Matter, C., Phillips, W. D., \& Alexander, K. Sensitization by annular surrounds: Sensitization and masking. Vision Research, 1971, 11, 1445-1458.

Tolnurst, D. J. Separate channels for the analysis of the shape and the movement of a moving visual stimulus. Journal of Physiology, 1973, 231, 385-402.

Tolmurst, D. J. Reaction times in the detection of gratings by human observers: A probabilistic mechanism. Vision Research, 1975, 15, 1143-1149. (a)

Tolhurst, D. J. Sustained and transient channels in human vision. Vision Research, 1975, 15, 1151-1155. (b)

Vassilev, A., \& Mitov, D. Perception time and spatial frequency. Vision Research, 1976, 16, 89-92.

Watson, A. B., \& Nachmias, J. Patterns of temporal interaction in the detection of gratings. Vision Research, 1977, 17, 893-902.

Watson, A. B., Thompson, P. G., Murphy, B. J., \& Nachmias, $\mathrm{J}$. Summation and discrimination of gratings moving in opposite directions. Vision Research, 1980, 20, 341-347.

WESTHEIMER, G. Spatial interaction in the human retina during scotopic vision. Journal of Physiology, 1965, 181, 881-894.

\section{NOTE}

1. It is to be noted that neither the previous experiments nor the present ones distinguish between spatial-frequency-specific and size-specific effects; such a discrimination would require studies with complex gratings.

Furthermore, the present experiments provide no data on the dependence of the magnitude and time course of masking on the spatial-frequency closeness between the stimuli. The strong masking effects at the 3:1 spatial-frequency ratio of the test and masking gratings seem to be unexpected in view of the data (Carter \& Henning, 1971; Stromeyer \& Julesz, 1972) on the spatialfrequency selectivity of masking. It should, however, be noted that the masking stimuli were of relatively high contrast and, according to the data of Legge and Foley (1979) and Sansbury (1974), this could cause their widespread effects. Whether such effects of suprathreshold stimuli are compatible with the hypothesis about the processing of the stimuli by narrow-tuned orientation- and spatial-frequency-selective mechanisms (Campbell \& Robson, 1968; Pantle \& Seculer, 1968) remains to be seen.

2. After this paper was submitted to the Publisher, Green (1981) published data which also demonstrated the importance of test stimulus spatial frequency for the time course of masking. Green's curves, obtained with fine test gratings $(7.8 \mathrm{c} / \mathrm{deg})$, are, however, pure sustained, unlike our mixed sustained-transient curves obtained at similar test frequencies. His data might also suggest that the test stimulus is the only determiner of the type of masking. In fact, the presence of a luminance step in Green's experiments makes the interpretation of masking effects criterion-dependent: increment threshold or contrast sensitivity functions have different shapes, as shown in his Figures 1 and 4.

(Received December 2, 1980; revision accepted for publication May 20, 1981.) 